\title{
Outcomes following gastrostomy: radiologically-inserted $v$. percutaneous endoscopic gastrostomy
}

\author{
J. S. Leeds ${ }^{1}$, M. E. McAlindon ${ }^{1}$, J. Grant ${ }^{1}$, H. E. Robson ${ }^{1}$, S. R. Morley ${ }^{2}$, F. K. T. Lee ${ }^{3}$ \\ and D. S. Sanders ${ }^{1}$ \\ ${ }^{1}$ Gastroenterology \& Liver Unit, ${ }^{2}$ Department of Clinical Chemistry and ${ }^{3}$ Department of Radiology, \\ Royal Hallamshire Hospital, Glossop Road, Sheffield S10 2JF, UK
}

Gastrostomy insertion has been demonstrated to be of benefit in selected patients ${ }^{(1,2)}$. Percutaneous endoscopic gastrostomy (PEG) using the pull-through technique is the most widely used insertion method, but it is recognised to have important complications, particularly in patients with respiratory risk factors. An alternative is a radiologically-inserted gastrostomy (RIG). It has been suggested that RIG may be advantageous in patients who are potentially at 'high risk' from respiratory complications. However, there are no large studies comparing PEG $v$. RIG.

All patients referred for a gastrostomy are prospectively included in a database along with demographic, biochemical and outcome data. Analysis of gastrostomy insertions over the period February 2004-February 2007 was performed with reference to method of insertion and outcome at $30 \mathrm{~d}$. Selection for method of insertion is left to the discretion of the referring clinician. Patients were allocated to the following subgroups: cognitive impairment $(n$ 5); dysphagic stroke $(n$ 36); nasopharygeal cancer $(n$ 175); neurological $(n$ 116); other (n 71).

Over the study period 170 RIG and 233 PEG were inserted (mean age 62 years, 268 males). There were no differences in age between the RIG group and PEG group and case mix was comparable except in the nasopharyngeal cancer group (proportionally more RIG). The RIG $30 \mathrm{~d}$ mortality was twenty-six of $170(15.3 \%)$ and the PEG $30 \mathrm{~d}$ mortality was twenty-five of $233(10.7 \% ; P=0.17)$. Mortality at 1 year was ninety-two of $170(54.1 \%)$ for RIG and 131 of $233(56.7 \%)$ for PEG $(P=0.60)$. Within subgroups the only significant difference in $30 \mathrm{~d}$ mortality was in those patients with nasopharyngeal cancer: fourteen of $106(13.2 \%)$ for RIG and one of 69 (1.4\%) for PEG $(P=0.005)$. However, patients referred for RIG were significantly older than those referred for PEG (mean age (years) $59.7 v$. 64 ; $P=0.019)$ and had a higher prevalence of significant comorbidities (21.1\% in the PEG group and $37.7 \%$ in the RIG group).

Overall, RIG and PEG appear to have similar $30 \mathrm{~d}$ mortality rates. In patients with nasopharyngeal cancer there was a higher mortality in those referred for RIG; however, pre-selection by the referring clinician as a result of perceived risk of endoscopic insertion may have biased the outcome. A randomized trial comparing both methods in this subgroup is needed.

1. Norton B, Homer-Ward M, Donnelly MT, Long RG \& Holmes GK (1996) A randomised prospective comparison of percutaneous endoscopic gastrostomy and naso-gastric tube feeding after acute dysphagic stroke. BMJ 312 (7022), 13-16.

2. Senft M \& Fietkau R (1993) The influence of supportive nutritional therapy via PEG on the quality of life of cancer patients. Support Care Cancer 1, 272-275. 